\title{
SOME HERMITE-HADAMARD TYPE INEQUALITIES VIA RIEMANN-LIOUVILLE FRACTIONAL CALCULUS
}

\author{
MARCELA V. MIHAI
}

\begin{abstract}
We provide some new Hermite-Hadamard type inequalities for functions whose derivatives in absolute value are convex, via Riemann-Liouville fractional integration.
\end{abstract}

\section{Introduction}

The Hermite-Hadamard inequality asserts that for every convex function $f: I \subseteq \mathbb{R} \rightarrow \mathbb{R}$ one has

$$
f\left(\frac{a+b}{2}\right) \leq \frac{1}{b-a} \int_{a}^{b} f(x) \mathrm{d} x \leq \frac{f(a)+f(b)}{2},
$$

where $a, b \in I$ with $a<b$. Both inequalities hold in reversed direction if $f$ is concave.

A remarkable variety of refinements and generalizations of Hermite-Hadamard inequality involving convex functions in the second sense have been found; see, for example, [1], [3], [5] and the references therein.

Our aim is to establish, using the Riemann-Liouville fractional calculus, some new Hermite-Hadamard type inequalities. We shall deal with functions whose derivatives in absolute value are convex.

Let $f \in L^{1}[a, b]$, where $a \geq 0$. The Riemann-Liouville integrals $J_{a+}^{\alpha} f$ and $J_{b-}^{\alpha} f$, of order $\alpha>0$, are defined by

and

$$
J_{a+}^{\alpha} f(x)=\frac{1}{\Gamma(\alpha)} \int_{a}^{x}(x-t)^{\alpha-1} f(t) \mathrm{d} t, \text { for } x>a
$$

$$
J_{b-}^{\alpha} f(x)=\frac{1}{\Gamma(\alpha)} \int_{x}^{b}(t-x)^{\alpha-1} f(t) \mathrm{d} t, \text { for } x<b,
$$

respectively. Here, $\Gamma(\alpha)=\int_{0}^{\infty} e^{-t} t^{\alpha-1} \mathrm{~d} t$ is the Gamma function. We also make the convention

$$
J_{a+}^{0} f(x)=J_{b-}^{0} f(x)=f(x) .
$$

More details about the Riemann-Liouville fractional integrals may be found in [2].

Received July 31, 2012, accepted December 06, 2012.

Communicated by Chung-Tsun Shieh.

2010 Mathematics Subject Classification. 26A51.

Key words and phrases. Convex function, Hermite-Hadamard inequality, Riemann-Liouville fractional integrals. 


\section{A lemma}

We assume throughout the present paper that $[a, b]$ is a subinterval of $[0, \infty)$ and $f$ : $[a, b] \rightarrow \mathbb{R}$ is a function differentiable on $(a, b)$ such that $f^{\prime} \in L^{1}[a, b]$. Before stating the results we establish the notation. Throughout this paper we denote the cumulative to the left $\alpha-$ gap by

$$
\begin{aligned}
\mathscr{L}_{\alpha}(a, b)= & \frac{1}{2}\left[f\left(\frac{3 a+b}{4}\right)+f\left(\frac{a+3 b}{4}\right)\right]-\frac{4^{\alpha-1} \Gamma(\alpha+1)}{(b-a)^{\alpha}}\left[J_{\frac{3 a+b}{4}-}^{\alpha} f(a)+J_{\frac{a+b}{2}-}^{\alpha} f\left(\frac{3 a+b}{4}\right)\right. \\
& \left.+J_{\frac{a+3 b}{4}-}^{\alpha} f\left(\frac{a+b}{2}\right)+J_{b-}^{\alpha} f\left(\frac{a+3 b}{4}\right)\right] .
\end{aligned}
$$

Thus, the particular case $\alpha=1$ gives

$$
\mathscr{L}(a, b)=\frac{1}{2}\left[f\left(\frac{3 a+b}{4}\right)+f\left(\frac{a+3 b}{4}\right)\right]-\frac{1}{b-a} \int_{a}^{b} f(t) \mathrm{d} t .
$$

In order to prove our main results we need the following lemma.

Lemma 1. We have

$$
\begin{aligned}
\mathscr{L}_{\alpha}(a, b)= & \frac{b-a}{16}\left[\int_{0}^{1} t^{\alpha} f^{\prime}\left(t \frac{3 a+b}{4}+(1-t) a\right) \mathrm{d} t+\int_{0}^{1}\left(t^{\alpha}-1\right) f^{\prime}\left(t \frac{a+b}{2}+(1-t) \frac{3 a+b}{4}\right) \mathrm{d} t\right. \\
& \left.+\int_{0}^{1} t^{\alpha} f^{\prime}\left(t \frac{a+3 b}{4}+(1-t) \frac{a+b}{2}\right) \mathrm{d} t+\int_{0}^{1}\left(t^{\alpha}-1\right) f^{\prime}\left(t b+(1-t) \frac{a+3 b}{4}\right) \mathrm{d} t\right] .
\end{aligned}
$$

Proof. We use the integration by parts and appropriate substitutions (such as $u=\frac{3 a+b}{4} t+$ $\left.a(1-t), v=\frac{a+b}{2} t+\frac{3 a+b}{4}(1-t), \ldots\right)$ to show that

$$
\begin{gathered}
\frac{b-a}{16} \int_{0}^{1} t^{\alpha} f^{\prime}\left(t \frac{3 a+b}{4}+(1-t) a\right) \mathrm{d} t=\frac{1}{4} f\left(\frac{3 a+b}{4}\right)-\frac{4^{\alpha-1} \Gamma(\alpha+1)}{(b-a)^{\alpha}} J_{\frac{3 a+b}{4}-}^{\alpha} f(a), \\
\frac{b-a}{16} \int_{0}^{1}\left(t^{\alpha}-1\right) f^{\prime}\left(t \frac{a+b}{2}+(1-t) \frac{3 a+b}{4}\right) \mathrm{d} t=\frac{1}{4} f\left(\frac{3 a+b}{4}\right)-\frac{4^{\alpha-1} \Gamma(\alpha+1)}{(b-a)^{\alpha}} J_{\frac{a+b}{2}-}^{\alpha} f\left(\frac{3 a+b}{4}\right), \\
\frac{b-a}{16} \int_{0}^{1} t^{\alpha} f^{\prime}\left(t \frac{a+3 b}{4}+(1-t) \frac{a+b}{2}\right) \mathrm{d} t=\frac{1}{4} f\left(\frac{a+3 b}{4}\right)-\frac{4^{\alpha-1} \Gamma(\alpha+1)}{(b-a)^{\alpha}} J_{\frac{a+3 b}{4}-}^{\alpha} f\left(\frac{a+b}{2}\right), \\
\frac{b-a}{16} \int_{0}^{1}\left(t^{\alpha}-1\right) f^{\prime}\left(t b+(1-t) \frac{a+3 b}{4}\right) \mathrm{d} t=\frac{1}{4} f\left(\frac{a+3 b}{4}\right)-\frac{4^{\alpha-1} \Gamma(\alpha+1)}{(b-a)^{\alpha}} J_{b-}^{\alpha} f\left(\frac{a+3 b}{4}\right) .
\end{gathered}
$$

The proof is completed.

\section{Inequalities of Hermite-Hadamard type}

We are now in a position to state and prove the following: 
Theorem 1. Assume $\left|f^{\prime}\right|$ is convex on $[a, b]$. Then

$$
\begin{aligned}
\left|\mathscr{L}_{\alpha}(a, b)\right| \leq & \frac{b-a}{32(\alpha+1)(\alpha+2)}\left[2\left|f^{\prime}(a)\right|+\left(\alpha^{2}+5 \alpha+2\right)\left|f^{\prime}\left(\frac{3 a+b}{4}\right)\right|\right. \\
& \left.+\left(\alpha^{2}+\alpha+2\right)\left|f^{\prime}\left(\frac{a+b}{2}\right)\right|+\left(\alpha^{2}+5 \alpha+2\right)\left|f^{\prime}\left(\frac{a+3 b}{4}\right)\right|+\alpha(\alpha+1)\left|f^{\prime}(b)\right|\right] .
\end{aligned}
$$

Proof. Using Lemma 1 and taking modulus, we infer from the convexity of $\left|f^{\prime}\right|$ that

$$
\begin{aligned}
\left|\mathscr{L}_{\alpha}(a, b)\right| \leq & \frac{b-a}{16}\left\{\int_{0}^{1} t^{\alpha}\left[t\left|f^{\prime}\left(\frac{3 a+b}{4}\right)\right|+(1-t)\left|f^{\prime}(a)\right|\right] \mathrm{d} t\right. \\
& +\int_{0}^{1}\left(1-t^{\alpha}\right)\left[t\left|f^{\prime}\left(\frac{a+b}{2}\right)\right|+(1-t)\left|f^{\prime}\left(\frac{3 a+b}{4}\right)\right|\right] \mathrm{d} t \\
& +\int_{0}^{1} t^{\alpha}\left[t\left|f^{\prime}\left(\frac{a+3 b}{4}\right)\right|+(1-t)\left|f^{\prime}\left(\frac{a+b}{2}\right)\right|\right] \mathrm{d} t \\
& \left.+\int_{0}^{1}\left(1-t^{\alpha}\right)\left[t\left|f^{\prime}(b)\right|+(1-t)\left|f^{\prime}\left(\frac{a+3 b}{4}\right)\right|\right] \mathrm{d} t\right\} .
\end{aligned}
$$

The result follows after a straightforward computation in the right hand side term. This ends the proof.

We recall that the Beta function (the Euler integral of the first kind), is defined by

$$
\mathrm{B}(x, y)=\int_{0}^{1} t^{x-1}(1-t)^{y-1} \mathrm{~d} t
$$

for $x, y>0$.

Our next results reads as:

Theorem 2. Assume $\left|f^{\prime}\right|^{q}$ is convex on $[a, b]$ for some fixed $q>1$. Then

$$
\begin{aligned}
\left|\mathscr{L}_{\alpha}(a, b)\right| \leq & \frac{b-a}{16}\left(\frac{1}{2}\right)^{1 / q}\left(\frac{1}{\alpha}\right)^{1 / p}\left\{\left(\frac{\alpha}{\alpha p+1}\right)^{1 / p}\left[\left|f^{\prime}\left(\frac{3 a+b}{4}\right)\right|^{q}+\left|f^{\prime}(a)\right|^{q}\right]^{1 / q}\right. \\
& +\left[\mathrm{B}\left(p+1, \frac{1}{\alpha}\right)\right]^{1 / p}\left[\left|f^{\prime}\left(\frac{a+b}{2}\right)\right|^{q}+\left|f^{\prime}\left(\frac{3 a+b}{4}\right)\right|^{q}\right]^{1 / q} \\
& +\left(\frac{\alpha}{\alpha p+1}\right)^{1 / p}\left[\left|f^{\prime}\left(\frac{a+3 b}{4}\right)\right|^{q}+\left|f^{\prime}\left(\frac{a+b}{2}\right)\right|^{q}\right]^{1 / q} \\
& \left.+\left[\mathrm{B}\left(p+1, \frac{1}{\alpha}\right)\right]^{1 / p}\left[\left|f^{\prime}\left(\frac{a+3 b}{4}\right)\right|^{q}+\left|f^{\prime}(b)\right|^{q}\right]^{1 / q}\right\},
\end{aligned}
$$

where $\frac{1}{p}+\frac{1}{q}=1$

We denote

$$
I_{1}=\int_{0}^{1}\left|f^{\prime}\left(t \frac{3 a+b}{4}+(1-t) a\right)\right|^{q} \mathrm{~d} t,
$$


and

$$
\begin{aligned}
& I_{2}=\int_{0}^{1}\left|f^{\prime}\left(t \frac{a+b}{2}+(1-t) \frac{3 a+b}{4}\right)\right|^{q} \mathrm{~d} t, \\
& I_{3}=\int_{0}^{1}\left|f^{\prime}\left(t \frac{a+3 b}{4}+(1-t) \frac{a+b}{2}\right)\right|^{q} \mathrm{~d} t
\end{aligned}
$$

$$
I_{4}=\int_{0}^{1}\left|f^{\prime}\left(t b+(1-t) \frac{a+3 b}{4}\right)\right|^{q} \mathrm{~d} t \text {. }
$$

Proof. According to Lemma 1 and Hölder's inequality, we have

$$
\begin{aligned}
\left|\mathscr{L}_{\alpha}(a, b)\right| \leq & \frac{b-a}{16}\left[\left(\int_{0}^{1}\left(t^{\alpha}\right)^{p} \mathrm{~d} t\right)^{1 / p}\left(\left(I_{1}\right)^{1 / q}+\left(I_{3}\right)^{1 / q}\right)\right. \\
& \left.+\left(\int_{0}^{1}\left(1-t^{\alpha}\right)^{p} \mathrm{~d} t\right)^{1 / p}\left(\left(I_{2}\right)^{1 / q}+\left(I_{4}\right)^{1 / q}\right)\right] .
\end{aligned}
$$

Here

$$
\begin{aligned}
I_{1} & \leq\left|f^{\prime}\left(\frac{3 a+b}{4}\right)\right|^{q} \int_{0}^{1} t \mathrm{~d} t+\left|f^{\prime}(a)\right|^{q} \int_{0}^{1}(1-t) \mathrm{d} t \\
& =\frac{1}{2}\left|f^{\prime}\left(\frac{3 a+b}{4}\right)\right|^{q}+\frac{1}{2}\left|f^{\prime}(a)\right|^{q}, \\
I_{2} & \leq \frac{1}{2}\left|f^{\prime}\left(\frac{a+b}{2}\right)\right|^{q}+\frac{1}{2}\left|f^{\prime}\left(\frac{3 a+b}{4}\right)\right|^{q}, \\
I_{3} & \leq \frac{1}{2}\left|f^{\prime}\left(\frac{a+3 b}{4}\right)\right|^{q}+\frac{1}{2}\left|f^{\prime}\left(\frac{a+b}{2}\right)\right|^{q}
\end{aligned}
$$

and

$$
I_{4} \leq \frac{1}{2}\left|f^{\prime}(b)\right|^{q}+\frac{1}{2}\left|f^{\prime}\left(\frac{a+3 b}{4}\right)\right|^{q} \text {. }
$$

These last inequalities hold due to the convexity of $\left|f^{\prime}\right|^{q}$ on $[a, b]$. The proof is complete.

Theorem 3. Assume $\left|f^{\prime}\right|^{q}$ is convex on $[a, b]$ for some fixed $q \geq 1$. Then the following inequality holds:

$$
\begin{aligned}
\left|\mathscr{L}_{\alpha}(a, b)\right| \leq & \left.\frac{b-a}{16}\left(\frac{1}{\alpha+1}\right)\right)^{1 / p}\left(\frac{1}{2(\alpha+1)(\alpha+2)}\right)^{1 / q} \\
& \times\left\{\left[2(\alpha+1)\left|f^{\prime}\left(\frac{3 a+b}{4}\right)\right|^{q}+2\left|f^{\prime}(a)\right|^{q}\right]^{1 / q}\right. \\
& +\alpha^{1 / p}\left[\alpha(\alpha+1)\left|f^{\prime}\left(\frac{a+b}{2}\right)\right|^{q}+\left(\alpha^{2}+3 \alpha\right)\left|f^{\prime}\left(\frac{3 a+b}{4}\right)\right|^{q}\right]^{1 / q} \\
& +\left[2(\alpha+1)\left|f^{\prime}\left(\frac{a+3 b}{4}\right)\right|^{q}+\left.2\left|f^{\prime}\left(\frac{a+b}{2}\right)\right|\right|^{q}\right]^{1 / q} \\
& \left.+\alpha^{1 / p}\left[\alpha(\alpha+1)\left|f^{\prime}(b)\right|^{q}+\left(\alpha^{2}+3 \alpha\right)\left|f^{\prime}\left(\frac{a+3 b}{4}\right)\right|^{q}\right]^{1 / q}\right\} .
\end{aligned}
$$


Proof. Using Lemma 1, the convexity of $\left|f^{\prime}\right|^{q}$ on $[a, b]$ and the power-mean inequality, we have

where

$$
\begin{aligned}
\left|\mathscr{L}_{\alpha}(a, b)\right| \leq & \frac{b-a}{16}\left[\left(\int_{0}^{1} t^{\alpha} \mathrm{d} t\right)^{1 / p}\left(\left(J_{1}\right)^{1 / q}+\left(J_{3}\right)^{1 / q}\right)\right. \\
& \left.+\left(\int_{0}^{1}\left(1-t^{\alpha}\right) \mathrm{d} t\right)^{1 / p}\left(\left(J_{2}\right)^{1 / q}+\left(J_{4}\right)^{1 / q}\right)\right],
\end{aligned}
$$

$$
\begin{aligned}
J_{1} & =\int_{0}^{1} t^{\alpha}\left|f^{\prime}\left(t \frac{3 a+b}{4}+(1-t) a\right)\right|^{q} \mathrm{~d} t \\
& \leq \frac{1}{\alpha+2}\left|f^{\prime}\left(\frac{3 a+b}{4}\right)\right|^{q}+\frac{1}{(\alpha+1)(\alpha+2)}\left|f^{\prime}(a)\right|^{q}, \\
J_{2} & =\int_{0}^{1}\left(1-t^{\alpha}\right)\left|f^{\prime}\left(t \frac{a+b}{2}+(1-t) \frac{3 a+b}{4}\right)\right|^{q} \mathrm{~d} t \\
& \leq \frac{\alpha}{2(\alpha+2)}\left|f^{\prime}\left(\frac{a+b}{2}\right)\right|^{q}+\frac{\alpha^{2}+3 \alpha}{2(\alpha+1)(\alpha+2)}\left|f^{\prime}\left(\frac{3 a+b}{4}\right)\right|^{q}, \\
J_{3} & =\int_{0}^{1} t^{\alpha}\left|f^{\prime}\left(t \frac{a+3 b}{4}+(1-t) \frac{a+b}{2}\right)\right|^{q} \mathrm{~d} t \\
& \leq \frac{1}{\alpha+2}\left|f^{\prime}\left(\frac{a+3 b}{4}\right)\right|^{q}+\frac{1}{(\alpha+1)(\alpha+2)}\left|f^{\prime}\left(\frac{a+b}{2}\right)\right|^{q}
\end{aligned}
$$

and

$$
\begin{aligned}
J_{4} & =\int_{0}^{1}\left(1-t^{\alpha}\right)\left|f^{\prime}\left(t b+(1-t) \frac{a+3 b}{4}\right)\right|^{q} \mathrm{~d} t \\
& \leq \frac{\alpha}{2(\alpha+2)}\left|f^{\prime}(b)\right|^{q}+\frac{\alpha^{2}+3 \alpha}{2(\alpha+1)(\alpha+2)}\left|f^{\prime}\left(\frac{a+3 b}{4}\right)\right|^{q} .
\end{aligned}
$$

Hence the proof of the theorem is complete.

Theorem 4. Assume $\left|f^{\prime}\right|^{q}$ is concave on $[a, b]$ for some fixed $q>1$. Then

$$
\begin{aligned}
\left|\mathscr{L}_{\alpha}(a, b)\right| \leq & \frac{b-a}{16}\left[\left(\frac{1}{\alpha p+1}\right)^{1 / p}\left|f^{\prime}\left(\frac{7 a+b}{8}\right)\right|+\left(\frac{1}{\alpha} \mathrm{B}\left(p+1, \frac{1}{\alpha}\right)\right)^{1 / p}\left|f^{\prime}\left(\frac{5 a+3 b}{8}\right)\right|\right. \\
& \left.+\left(\frac{1}{\alpha p+1}\right)^{1 / p}\left|f^{\prime}\left(\frac{3 a+5 b}{8}\right)\right|+\left(\frac{1}{\alpha} \mathrm{B}\left(p+1, \frac{1}{\alpha}\right)\right)^{1 / p}\left|f^{\prime}\left(\frac{a+7 b}{8}\right)\right|\right\},
\end{aligned}
$$

where $\frac{1}{p}+\frac{1}{q}=1$.

Proof. From Lemma 1 and Hölder's integral inequality for $q>1$ and $p=\frac{q}{q-1}$, we have

$$
\begin{aligned}
\left|\mathscr{L}_{\alpha}(a, b)\right| \leq & \frac{b-a}{16}\left[\left(\int_{0}^{1}\left(t^{\alpha}\right)^{p} \mathrm{~d} t\right)^{1 / p}\left(\left(I_{1}\right)^{1 / q}+\left(I_{3}\right)^{1 / q}\right)\right. \\
& \left.+\left(\int_{0}^{1}\left(1-t^{\alpha}\right)^{p} \mathrm{~d} t\right)^{1 / p}\left(\left(I_{2}\right)^{1 / q}+\left(I_{4}\right)^{1 / q}\right)\right]
\end{aligned}
$$


for all $x \in[a, b]$, where

and

$$
\begin{aligned}
& I_{1} \leq\left|f^{\prime}\left(\frac{\frac{3 a+b}{4}+a}{2}\right)\right|^{q}=\left|f^{\prime}\left(\frac{7 a+b}{8}\right)\right|^{q}, \\
& I_{2} \leq\left|f^{\prime}\left(\frac{5 a+3 b}{8}\right)\right|^{q}, \\
& I_{3} \leq\left|f^{\prime}\left(\frac{3 a+5 b}{8}\right)\right|^{q}
\end{aligned}
$$

$$
I_{4} \leq\left|f^{\prime}\left(\frac{a+7 b}{8}\right)\right|^{q} .
$$

We used the concavity of $\left|f^{\prime}\right|^{q}$ on $[a, b]$ and the inequality (1.1) in order to obtain the last four inequalities. This completes the proof of the theorem.

\section{Remarks}

Remark 1. For $\alpha=1$ in the Theorems 1, 2, 3, respectively 4, we recover the results stated in ([4, Theorems 1-4]). Also for $\alpha=1$ in Lemma 1, we get ([4, Lemma 1]).

Remark 2. By considering the cumulative to the right $\alpha$-gap defined as

$$
\begin{aligned}
\mathscr{R}_{\alpha}(a, b)= & -\frac{1}{2}\left[\frac{f(a)+f(b)}{2}+f\left(\frac{a+b}{2}\right)\right]+\frac{4^{\alpha-1} \Gamma(\alpha+1)}{(b-a)^{\alpha}}\left[J_{a+}^{\alpha} f\left(\frac{3 a+b}{4}\right)+J_{\frac{3 a+b}{4}+}^{\alpha} f\left(\frac{a+b}{2}\right)\right. \\
& \left.+J_{\frac{a+b}{2}+}^{\alpha} f\left(\frac{a+3 b}{4}\right)+J_{\frac{a+3 b}{4}+}^{\alpha} f(b)\right],
\end{aligned}
$$

one can obtain similar results. However, this is not the purpose of the present paper.

\section{References}

[1] S. S. Dragomir, C. E. M. Pearce, Selected Topic on Hermite-Hadamard Inequalities and Applications, Melbourne and Adelaide, December, 2000.

[2] R. Gorenflo, F. Mainardi, Fractional Calculus: Integral and Differential Equations of Fractional Order, Springer Verlag, Wien, 1997.

[3] H. Kavurmaci, M. Avci, M. E. Özdemir, New inequalities of Hermite-Hadamard type for convex functions with applications, arXiv: 1006.1593v1[math. CA].

[4] M. A. Latif, New inequalities of Hermite-Hadamard type for functions whose derivatives in absolute value are convex with applications, RGMIA Research Report Collection, 15 (2012), Article 34, 13 pp.

[5] C. P. Niculescu, L.-E. Persson, Convex Functions and their Applications. A Contemporary Approach. CMS Books in Mathematics vol. 23, Springer-Verlag, New York, 2006. 